\title{
Die Elimination der Masern in der Schweiz
}

\section{Christoph Bergera, Barbara Weil ${ }^{b}$}

a Co-Leiter Abteilung Infektiologie und Spitalhygiene, Universitäts-Kinderkliniken, Zürich

b Leiterin Abteilung Gesundheitsförderung und Prävention, FMH
Korrespondenz:

Prof. Dr. med. Christoph Berger Co-Leiter Abteilung Infektiologie und Spitalhygiene

Universitäts-Kinderkliniken

Steinwiesstrasse 75

CH-8032 Zürich

Tel. 0442667840

Fax 0442668072

christoph.berger[at]kispi.uzh.ch
Die Masern sind - dank Impfungen - selten geworden. Masernviren kommen nur beim Menschen vor und werden sehr rasch via Husten und Niesen übertragen. Weltweit kommt die Krankheit heute vorwiegend noch in Asien, Afrika und einigen Ländern Europas mit einer zu niedrigen Impfrate vor - wie etwa der Schweiz. Mit über 4400 gemeldeten Erkrankungen, Hunderten von Hospitalisationen und Kosten von über 15 Millionen Schweizer Franken erlebte die Schweiz von 2006 bis 2009 eine Epidemie. 2009 starb ein zuvor gesundes Mädchen an Komplikationen der Masern. Würde hierzulande überhaupt nicht gegen Masern geimpft, käme es jedes Jahr zu etwa 70000 Erkrankungen und 20 bis 30 Maserntodesfällen.

Was bedeutet «Elimination»? In der Schweiz konnten dank Impfungen bereits die Kinderlähmung (Polio) sowie Pocken eliminiert werden; bei den Pocken gelang dies 1981 weltweit. Die Masern gelten in einem Land oder einem Kontinent dann als eliminiert, wenn jährlich maximal ein Fall pro eine Million Einwohner auftritt. Dazu müssen 95\% der Bevölkerung immun, das heisst, zweimal gegen Masern geimpft sein oder die Krankheit durchlebt haben.

Bei einer Durchimpfung von 95\% entsteht eine sogenannte Herdenimmunität; die Masernviren können sich nicht mehr verbreiten und verschwinden aus einer Population. Mehrere Länder Skandinaviens, Australien sowie ganz Nord- und Südamerika zeigen, dass dies möglich ist: Sie sind heute dank der Impfung praktisch masernfrei. Davon ist die Schweiz

\section{Abbildung 1}

Masernexanthem am 3. Tag. (Bild: CDC - Centers of Disease Control and Prevention / Dr. Heinz F. Eichenwald)

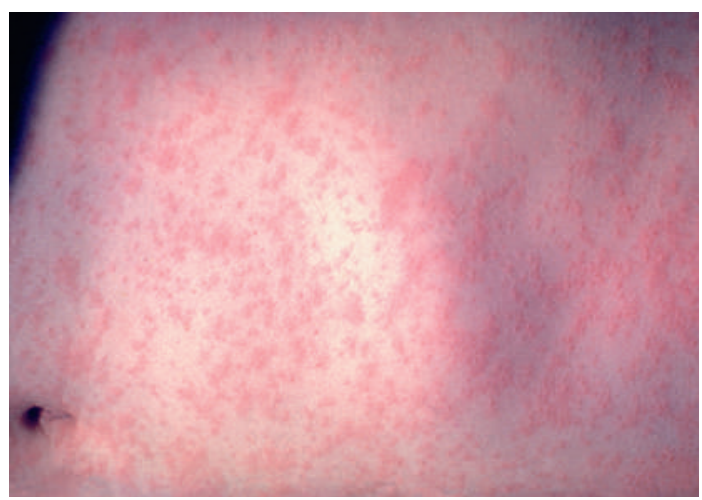

noch weit entfernt, denn zurzeit sind erst $86 \%$ der Zweijährigen mit 2 Impfdosen vor Masern geschützt. Die Durchimpfung variiert stark von Kanton zu Kanton, Spitzenreiter 2010 war der Kanton Genf mit 93\%.

Masern werden immer wieder als «harmlose Kinderkrankheit» bezeichnet. Doch sie sind weder harmlos noch eine reine Kinderkrankheit. Erkranken können auch Erwachsene. Die erste Krankheitsphase beginnt 7 bis 18 Tage nach Ansteckung mit grippalen Symptomen und oft einer Konjunktivitis. Gleichzeitig sind die Infizierten bereits kontagiös! Viele Erkrankte verkennen diese Vorsymptome als «Erkältung». Ohne Vorsichtsmassnahmen stecken sie so ungeimpfte Menschen in ihrem Umfeld an, wodurch sich Masern rasch ausbreiten können. Erst nach weiteren vier Tagen folgt die zweite Phase mit hohem Fieber, Appetitlosigkeit, starkem Krankheitsgefühl, Bettlägerigkeit und dem typischen Exanthem (Abb. 1 und 2).

Masern können schwere Komplikationen verursachen, die bei ca.10\% der Erkrankten eine Hospitalisierung erfordern und tödlich verlaufen können. Diese Komplikationen - und nicht etwa der harmlose Hautausschlag - sind der Grund für die Impfung. In etwa $10 \%$ treten eine Otitis media, eine Bronchotracheitis (croup) oder Fieberkrämpfe auf, in etwa 5\% eine Pneumonie. Einer von tausend Masernkranken erleidet eine Enzephalitis. Eine durchlebte Erkrankung stärkt keineswegs das Immunsystem, im Gegenteil: Sie führt zu einer Leukopenie und einer über Wochen dauernden Immunsuppression. Sehr selten kommt es Jahre nach einer Erkrankung als Spätkomplikation zu einer stets tödlich verlaufenden subakuten, sklerosierenden Panenzephalitis (SSPE).

Die Masernimpfung ist in der Schweiz seit 1976 empfohlen, die kombinierte Impfung gegen Masern, Röteln und Mumps (MMR) seit 1985. Die zurzeit gültige Empfehlung: eine MMR-Dosis im Alter von zwölf Monaten, eine zweite im Alter von 15 bis 24 Monaten. Nachholimpfungen sind in jedem Alter möglich und werden allen nicht-immunen Personen empfohlen, die 1964 oder später geboren wurden. Zwei Dosen MMR bieten einen meist lebenslang anhaltenden Schutz gegen diese Krankheiten. Mit der kombinierten Impfung gegen Masern, Mumps und Röteln (MMR) werden auch die Röteln zum Verschwinden gebracht.

Die Impfung ist sehr gut verträglich, weltweit 


\section{Abbildung 2}

Zeitlicher Verlauf einer Masernerkrankung und der Masernübertragung. Für die Massnahmen entscheidend ist der Exanthembeginn am Tag null. (Quelle: Kantonsärztl. Dienst Genf; adaptiert)

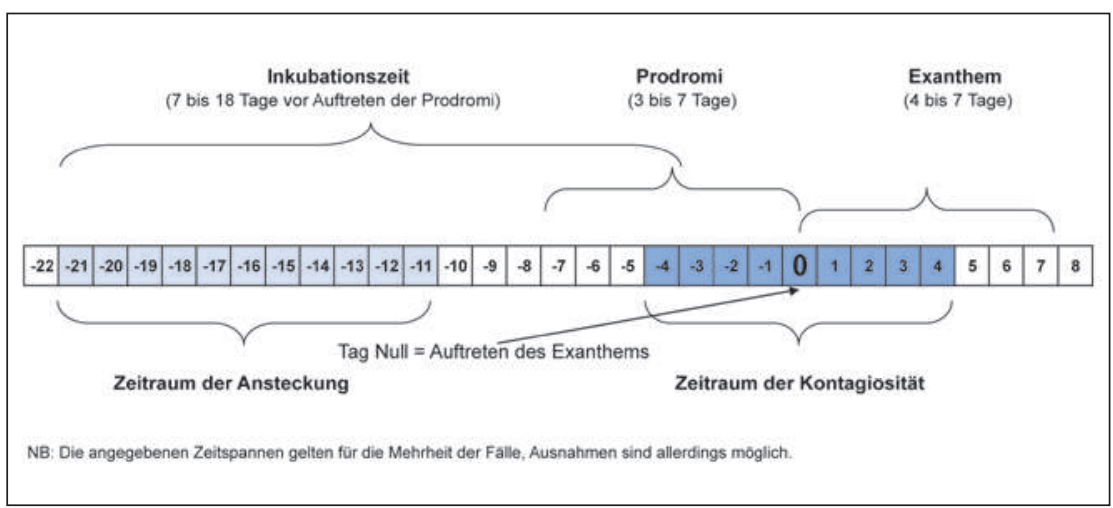

haben seit den 70er Jahren mehrere Milliarden Kinder und Erwachsene eine Masern- oder MMR-Impfung erhalten. Als Nebenwirkung kann ein leichter, harmloser Hautausschlag auftreten, ein Fieberkrampf tritt in weniger als $0,03 \%$ auf, allergische Systemreaktionen in weniger als 0,001\%. Bei Frauen, die sich erst nach der Pubertät impfen lassen, treten gelegentlich vorübergehende Gelenkschmerzen auf. Wichtigste Kontraindikationen sind eine bekannte Schwangerschaft sowie eine schwere Immunschwäche.

\section{Die nationale Maserneliminationsstrategie 2011-2015}

«Europa masernfrei bis Ende 2015» heisst das Ziel der Mitgliedstaaten der europäischen Region der Weltgesundheitsorganisation (WHO), zu der auch die Schweiz gehört. Dazu wurde die nationale Maserneliminationsstrategie 2011-2015 gemeinsam von Bund, Kantonen, verschiedenen medizinischen Fachgesellschaften und Organisationen des Gesundheitswesens erarbeitet. Der Bundesrat hat sie im Dezember 2011 zur nationalen Umsetzung verabschiedet.

Verschiedene Massnahmen tragen zur Zielerreichung bei, unter anderem wird sich ein «Masernkomitee» für die breite politische und kommunikative Abstützung der Masernelimination einsetzen. Ferner soll erreicht werden, dass 95\% der zweijährigen Kinder mit zwei Impfdosen vor den Masern geschützt sind. Kindertagesstätten (Kitas) sollen möglichst mit einer betreuenden Ärztin oder einem Arzt zusammenarbeiten. Eltern sollen besser über Masern, deren Komplikationen sowie die Impfung Bescheid wissen und frühzeitig darüber informiert sein, dass ungeimpfte Kinder im Falle eines Masernausbruchs vorübergehend aus der Kita oder Schule ausgeschlossen werden. Weiter beinhaltet die Strategie einen einfachen Zugang und niederschwellige Angebote zur Nachholimpfung, und möglichst jeder Arzt-Patienten-Kontakt soll dazu genutzt werden, den Impfstatus zu überprüfen und
Stand April 2013, www.bag.admin.ch/masern
Richtlinien zur Bekämpfung von Masern und Masernausbrüchen, Bundesamt für bei Bedarf Nachholimpfungen anzubieten. Dank einer Franchisenbefreiung der Masern- und MMRNachholimpfung von 2013 bis 2015 muss nur noch der Selbstbehalt bezahlt werden. Die Befreiung beinhaltet die Konsultation, den Impfstoff und die Impfung. Mit einer von Bund und Kantonen entwickelten nationalen Informations-Kampagne sollen Jugendliche und Erwachsene unter 50 Jahren zum Thema Masern und Masernimpfung sensibilisiert und Nichtimmune zur Nachholimpfung motiviert werden. Notabene sind und bleiben Impfungen in der Schweiz freiwillig.

Neu kann man seine eigenen Impfungen auch auf der Webseite www.meineimpfungen.ch eingeben, so seinen Impfstatus überprüfen lassen und die Information erhalten, ob zusätzliche Impfungen oder Auffrischimpfungen gemäss Schweizer Impfplan empfohlen sind.

Schliesslich soll die epidemiologische Überwachung der Masern verbessert werden. Bereits bei einem Verdacht sollen Masernfälle rasch erkannt, an den kantonsärztlichen Dienst gemeldet und im Labor bestätigt werden. Nur so haben die Massnahmen zur Ausbruchskontrolle Erfolg. Masernausbrüche sollen in allen Kantonen rasch und einheitlich bekämpft werden*. Dies funktioniert in einigen Kantonen wie zum Beispiel in Genf bereits sehr gut, wodurch 2011 eine Epidemie verhindert werden konnte.

Ärztinnen und Ärzte spielen bei der Information, der Motivation und der Verbesserung der Durchimpfung eine wichtige Rolle. Sie haben grossen Einfluss auf das Impfverhalten ihrer Patientinnen und Patienten. Auch können sich «Noch-nicht-Immune» selbst mit zwei Dosen vor einer Masernerkrankung schützen, mit gutem Beispiel vorangehen und zur Verhütung von Infektionen in der Praxis und im Spital beitragen.

Weiter sollten Ärztinnen und Ärzte an Masern und an die MMR-Nachholimpfung «denken» und beispielsweise bei einer Tetanus-Auffrischimpfung, bei gynäkologischen Kontrollen oder bei reisemedizinischen Beratungen vor Auslandsreisen auch den Masern-Impfstatus überprüfen, offen über Risiken und Vorteile der Impfung sprechen, auf Fragen eingehen und Nichtimmune für die MMR-Impfung motivieren.

Viele Arztpraxen laden die Eltern von Kleinkindern zum empfohlenen Zeitpunkt zur MMR-Impfung ein. Dafür stehen auch technische Hilfsmittel wie etwa das System Viavac (www.viavac.ch / www. meineimpfungen.ch) zur Verfügung.

Ärztinnen und Ärzte sollen jeden Masernverdacht (Trias: 1. Fieber, 2. makulopapulöses Exanthem, 3. Husten, Rhinitis oder Konjunktivitis) unverzüglich dem Kantonsärztlichen Dienst melden und eine Laboruntersuchung veranlassen.

Weitere Informationen unter: www.bag.admin. ch/masern 